\title{
An Audit of Post-0perative Hospital Stay and Complications of THR in a Tertiary Care Hospital
}

ISSN: 2576-8875

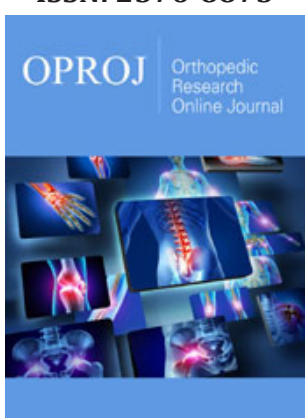

*Corresponding author: Muhammad Inam, Department of Orthopedic and Trauma, Medical Teaching Institute, Lady Reading Hospital Peshawar, Pakistan

Submission: 制 March 12, 2021

Published: 疅 March 22, 2021

Volume 8 - Issue 1

How to cite this article: Muhammad Inam, Ihsanullah, Javed Iqbal, Muhammad Shabir, Mian Amjad Ali. An Audit of PostOperative Hospital Stay and Complications of THR in a Tertiary Care Hospital. Ortho Res Online J. 8(1). OPROJ. 000680. 2021. DOI: $10.31031 /$ OPROJ.2021.08.000680

Copyright@: Muhammad Inam, This article is distributed under the terms of the Creative Commons Attribution 4.0 International License, which permits unrestricted use and redistribution provided that the original author and source are credited.

\author{
Muhammad Inam ${ }^{1 *}$, Ihsanullah ${ }^{2}$, Javed Iqbal ${ }^{3}$, Muhammad Shabir ${ }^{1}$ and Mian \\ Amjad Ali \\ ${ }^{1}$ Department of Orthopedic and Trauma, Medical Teaching Institute, Lady Reading Hospital \\ Peshawar, Pakistan \\ ${ }^{2}$ District Headquarter Hospital, Pakistan \\ ${ }^{3}$ Medical Officer, Tehsil Headquarter Hospital, Pakistan
}

\begin{abstract}
Objective: The objective of this study is to determine post-operative hospital stay and complications of total hip replacement in a tertiary care hospital.
\end{abstract}

Material and Method: This Descriptive study was conducted in the Department of Orthopedic and Trauma, Medical Teaching Institute Lady Reading Hospital Peshawar from March 2017 to February 2018 on 39 consecutive patient of age 55 and above with primary osteoarthritis of the hip. In all these patients cemented arthroplasty of the same manufacturer was done through direct lateral approach and patients were mobilized on first post-operative day. Risk Assessment and Prediction Tool (RAPT) Score was calculated pre-operatively for every patient and hospital stay was arranged accordingly to avoid any complications.

Results: Out of thirty-nine patients, $13(33.3 \%)$ were female and 26(66.7\%) were male. Mean age of the patients was 63.05(Std. Deviation 4.359) with minimum 55 years and maximum 70 years. Left side was affected in 17 (43.6\%) patients, while right side was affected in 22(56.4\%) patients. Pre-operatively the Risk Assessment and Prediction Tool (RAPT) Score was less than six score in 3(7.7\%), score between 6-9 in $16(41.0 \%)$ and score more than 9 in $20(51.3 \%)$ patients. Average Post-operative hospital stay was 4.28 with minimum of 2 days and maximum of 7 days with standard deviation of 1.654 . Eleven $(28.2 \%)$ patients have three days post-operative hospital stay, $8(20.5 \%)$ has five days and $7(17.9 \%)$ has six days post-operative hospital stay. The calculated predicted value in our study was $97.4 \%$

Conclusion: Preoperative assessment of the patients undergoing Total Hip Replacement not only reduces complication rates but also reduces socioeconomic burden on hospital as well as on patients.

Keywords: Arthroplasty; Assessment; Hip; Hospitalization; Inpatient; Replacement

\section{Introduction}

In old age the quality of bone is poor and usually fracture occurs in load bearing areas which is called fragility facture [1]. Not only bone fracture in old age but also the Joints also became weak and painful which's known as or Osteoarthritis. In both extreme the patient needs some intervention to reduce his pain and make him mobile [2]. If fracture of hip is extracapsular then fixation is needed which may be done by different methods like dynamic hip screw while intracapsular fracture needs some sort of arthroplasty [3]. Arthroplasty may be either hemi or total. It may be either cemented or uncemented. In osteoarthritis of the hip joint the only option that is used worldwide is arthroplasty. Most of the arthroplasty in this part of the world is cemented in old age. Arthroplasty or joint replacement is best option in old age due to fracture neck of femur or osteoarthritis, but it is not free of complications. Patients needs to be educated preoperatively [4]. Lifestyle modification is very important for a successful total hip replacement. If the patient does not follow the instruction it may dislocate. In our part of the world most of the people are uneducated and they dislocate their joint very easily [5]. There is operative option to reduce joint dislocation like approach, dual mobility joint and constrained joint. The surgeon tries to use the best approach to avoid dislocation and are more meticulous about closure of the joint [6]. The dual mobility and constrained joints are 
expensive, and the patient cannot afford it. So conventional joint is used most of time [7]. If the patients are well determined and follow the instruction, then dislocation changes are negligible [8]. There are certain tools that has to be used preoperatively and score it for prediction of post-operative hospitalization of patients to reduce the complication rate [9]. One of that tools is known as Risk Assessment and Prediction Tool (RAPT) $[10,11]$. This tool is scored preoperatively to predict any complication and to act accordingly. THR is a major surgery and most of time patient bear all the cost of the implants. Without knowing the tentative complication and hospital cost in terms of hospital stay make a big problem postoperatively for the patient. This study has shown us about the possible complication and hospital stay postoperatively in THR. The objective of this study is to determine post-operative hospital stay and complications of total hip replacement in a tertiary care hospital.

\section{Material and Method}

This Descriptive study was conducted in the Department of Orthopedic and Trauma, Medical Teaching Institute Lady Reading
Hospital Peshawar from March 2017 to February 2018 on 39 consecutive patient of age 55 and above with primary osteoarthritis of the hip. All those patients of either gender that have primary osteoarthritis of hip, diagnosed clinically and confirmed by radiograph of the hip taken in anteroposterior and lateral projection. Then patients were counseled about the procedure and postoperative protocol for daily living activities. That patient who was willing to be put in the study were included in the study with written informed consent. All the co morbid conditions were optimized before operation.

Preoperative all the patients were evaluated with Risk Assessment and Prediction Tool (RAPT) and categorized in high risk, medium risk and low risk group. RAPT includes age of the patient, gender, gait aid used, helper needed and living alone or with others. Total score is 12 , if the score is less than six (High Risk Group) then extended hospitalization is needed, if the score is between 6 and 9(Medium Risk Group) then rehabilitation is needed at home while if the score is more than 9(Low Risk Group) then early discharge without any rehabilitation is need (Figure 1).

\begin{tabular}{|c|c|c|}
\hline RISK ASSESSMENT AND PREDI & CTION TOOL & \\
\hline Score & & \\
\hline 1. What is your age group? & $50-65$ years & $=2$ \\
\hline & $66-75$ years & $=1$ \\
\hline & $>75$ years & $=0$ \\
\hline 2. Gender? & Male & $=2$ \\
\hline & Female & $=1$ \\
\hline 3. How far on average can you & Two blocks or more (+/-rest) & $=2$ \\
\hline walk? & $1-2$ blocks ( + --rest) & $=1$ \\
\hline (a block is 200 metres) & Housebound (most of time) & $=0$ \\
\hline 4. Which gait aid do you use? & None & $=2$ \\
\hline (more often than not) & Single-point stick & $=1$ \\
\hline & Crutches/frame & $=0$ \\
\hline 5. Do you use community & None or one per week & $=1$ \\
\hline supports? & Two or more per week & $=0$ \\
\hline $\begin{array}{l}\text { (home help, meals on wheels, } \\
\text { district nursing) }\end{array}$ & & \\
\hline 6. Will you live with someone & Yes & $=3$ \\
\hline $\begin{array}{l}\text { who can care for you after your } \\
\text { operation? }\end{array}$ & No & $=0$ \\
\hline Your score (out of 12) & & \\
\hline $\begin{array}{l}\text { Scores }<6 \text { - Extended inpatient } \\
\text { Score } 6-9 \text {-Additional interven } \\
\text { in the home) }\end{array}$ & $\begin{array}{l}\text { rehabilitation } \\
\text { tion to discharge directly home ( }\end{array}$ & Rehabilitation \\
\hline Score $>9$ - Directly home. & & \\
\hline
\end{tabular}

Figure 1: 
Risk Assessment and Prediction Tool (RAPT) was filled by the patients, scored and evaluated before surgery to predict the hospitalization days to avoid postoperative complication. Hip was operated with direct lateral approach and cemented arthroplasty was done and the implants used in all cases were from the same manufacturer. After closure, a pillow was put to the outer side of limb to avoid external rotation and hip flexion for 24 hours, and prophylactic intravenous antibiotics were given for five days. Vancomycin 2 grams and Cefoperazone + sulbactum 2 grams were given intra operatively 20 minutes before giving incision. The same antibiotics were given for first twenty-four hours and then the vancomycin was stopped. Anterior posterior and lateral radiograph were taken to see the position of cup and stem. On first post-operative day the patient is mobilized by the physiotherapist and walking is allowed with the help of Zimmer frame to avoid fall. The patients were instructed to sleep on operated side, use western commode in toilet, use a stick to avoid fall, use of wall mounted handles in toilets, and do not flex, abduct or adduct hip more than $90^{\circ}$.

All the data collected with the help of a proforma was then put into the SPSS version 17 and analyzed for frequencies.

\section{Results}

A total of fifty-four patients were included in the study but at final follow up only thirty-nine has been seen. So, we analyzed only those thirty-nine patients for any complication. Out of thirty-nine patients, $13(33.3 \%)$ were female and $26(66.7 \%)$ were male (Table
1). Mean age of the patients was 63.05(Std. Deviation 4.359) with minimum 55 years and maximum 70 years (Table 2). Left side was affected in 17 (43.6\%) patients, while right side was affected in $22(56.4 \%)$ patients (Table 3). For ease we have divided the RAPT score into high-risk group (Score less than 6), medium risk group (Score 6-9) and low risk group (Score more than 9). Preoperatively the Risk Assessment and Prediction Tool (RAPT) Score was less than six score in 3(7.7\%), score between 6-9 in 16(41.0\%) and score more than 9 in 20(51.3\%) patients (Table 4). Average post-operative hospital stay was 4.28 with minimum of 2 days and maximum of 7 days with standard deviation of 1.654 (Table 2). According to the RAPT score $3(7.7 \%)$ needs prolonged hospital stay, $16(41.0 \%)$ needed discharge from hospital with rehabilitation at home and 20(51.3\%) needed discharge direct to home while post operatively only $2(5.1 \%)$ patients has needed prolong stay due to complications. Eleven (28.2\%) patients have three days postoperative hospital stay, $8(20.5 \%)$ has five days, $7(17.9 \%)$ has six days while $4(10.3 \%)$ patients have 7 days of post-operative hospital stay (Table 5). There were two cases of surgical site infection. In one patient it was a superficial infection and was treated with injection cefoperazone + sulbactam 2gram intravenously for 14 days and then switched to oral Co-amoxiclav 1 gram twice daily for one month. The other case with infection was managed with debridement and same antibiotics. These two patients had a prolong hospital stay as compare to other. If we put these two cases in high-risk group then the predicted value of RAPT score was $97.4 \%$.

Table 1:

\begin{tabular}{|c|c|c|c|c|c|}
\hline \multicolumn{9}{|c|}{ Gender of Patient } \\
\hline \multirow{3}{*}{ Valid } & Female & Frequency & Percent & Valid Percent & Cumulative Percent \\
\cline { 2 - 7 } & Male & 13 & 33.3 & 33.3 & 33.3 \\
\cline { 2 - 7 } & Total & 26 & 66.7 & 66.7 & 100 \\
\hline
\end{tabular}

Table 2:

\begin{tabular}{|c|c|c|c|c|}
\hline \multicolumn{5}{|c|}{ Statistics } \\
\hline & & Post Operative Stay & Age of the Patient & Risk Assessment and Prediction Tool \\
\hline \multirow{10}{*}{$\mathrm{N}$} & Valid & 39 & 39 & 39 \\
\hline & Missing & 0 & 0 & 0 \\
\hline & Mean & 4.28 & 63.05 & 2.44 \\
\hline & Std. Error of Mean & 0.265 & 0.698 & 0.103 \\
\hline & Median & 4 & 64 & 3 \\
\hline & Mode & 3 & $60 \mathrm{a}$ & 3 \\
\hline & Std. Deviation & 1.654 & 4.359 & 0.641 \\
\hline & Range & 5 & 15 & 2 \\
\hline & Minimum & 2 & 55 & 1 \\
\hline & Maximum & 7 & 70 & 3 \\
\hline
\end{tabular}


Table 3:

\begin{tabular}{|c|c|c|c|c|c|}
\hline \multicolumn{6}{|c|}{ Side of Involvement } \\
\hline \multirow{5}{*}{ Valid } & & Frequency & Percent & Valid & Cumulative \\
\hline & & & & Percent & Percent \\
\hline & Left & 17 & 43.6 & 43.6 & 43.6 \\
\hline & Right & 22 & 56.4 & 56.4 & 100 \\
\hline & Total & 39 & 100 & 100 & \\
\hline
\end{tabular}

Table 4:

\begin{tabular}{|c|c|c|c|c|c|}
\hline \multicolumn{7}{|c|}{ Risk Assessment and Prediction Tool Score of the Patients } \\
\hline Group & Score & Frequency & Percent & Valid Percent & Cumulative Percent \\
\hline 1. High risk & Score Less than 6 & 3 & 7.7 & 7.7 & 7.7 \\
\hline 2. Medium risk & Score between 6 and 9 & 16 & 41 & 41 & 48.7 \\
\hline 3. Low risk & Score more than 9 & 20 & 51.3 & 51.3 & 100 \\
\hline
\end{tabular}

Table 5:

\begin{tabular}{|c|c|c|c|}
\hline \multicolumn{4}{|c|}{ Hospital Stay } \\
\hline Days & Frequency & Percent & Valid Percent \\
\hline 2 & 6 & 15.4 & 15.4 \\
\hline 3 & 11 & 28.2 & 28.2 \\
\hline 4 & 3 & 7.7 & 7.7 \\
\hline 5 & 8 & 20.5 & 20.5 \\
\hline 6 & 7 & 17.9 & 17.9 \\
\hline 7 & 4 & 10.3 & 10.3 \\
\hline Total & 39 & 100 & 100 \\
\hline
\end{tabular}

\section{Discussion}

Most of the hospital try to reduce the cost of hospital by cutting short of the hospital stay either preoperative of postoperative or both. As most of the economic burden is on the patients for arthroplasties, we have tried to reduce the such cost by reducing length of hospital stay that not only has helped in economy but also has minimized complication like cross infection, so we started using RAPT in our department for hip arthroplasty [3,5-7,10].

Australian orthopedist has originally developed the RAPT which is aimed to predict discharge of patients in Arthroplasties preoperatively. This allows the entire caregivers of the department to optimize the patient's care according to the needs [11]. A study done by Abbas et al. [12] has reported complication rate of $19.6 \%$ (39) in which the dislocation was most common 13 patients $(6.5 \%)$, while infection occurred in $4(2 \%)$ of patient that all needed some intervention. They have not used RAPT that's why their complication rate is high. In their study many minor complications also occurred that were managed conservatively like; surgical site infection in $2.5 \%$, urinary tract infection in $2.5 \%$, pleural effusion and pneumonia in $2 \%$, deep venous thrombosis in $0.5 \%$ and myocardial infarction in $0.5 \%$.
Hansen et al. [13] in 2015, studied 3,213 patients with arthroplasties and showed that RAPT score has an accurate prediction for patients discharge disposition. He found that there was $78 \%$ predictive accuracy overall but for hip it was $80 \%$. The predictive accuracy of RAPT was more than $90 \%$ of the score of $<6$ that has been sent to inpatient rehabilitation center and score $>10$ that has been sent to home, while it was lowest for scores between 7 and 10 which is almost comparable to our study. On the base of such finding he categorized his patients into low risk $>10$, intermediate risk 7 to 10 and high risk $<7$ of RAPT score. In current study high risk patients were $3(7.7 \%)$, intermediate risk was $16(41.0 \%)$ and low risk patients were $20(51.3 \%)$.

Bozic et al. [14] has compared the hospital cost for primary versus revision arthroplasties in term of in hospital stay and found that revision arthroplasties have higher cost and in patient stay. In his study the average complication rate was 32\% higher and the average inpatient stay was $16 \%$ longer than the primary arthroplasty. He concludes that preoperative risk assessment for arthroplasty can minimize higher resource utilization of the hospital and as well as complication rate.

Bozic et al. [15] studied 7818 patients for discharge to inpatient rehabilitation center after joint replacement evaluated by RAPT score. In his study $29 \%$ of high-risk patients that have score of less than six were discharged to inpatient rehabilitation center after surgery. The patient has high risk and was send to inpatient rehabilitation center was Old age, higher ASA grade, insured patients, and female gender. In our study there was only $7.7 \%$ patients that need inpatient rehabilitation.

Another study done by Tan et al. [16] in 2014 on 569 patient in which he pointed out that RAPT score has high significance for predicting the hospital stay following arthroplasty $(R=0.24, P<0.001)$; the shorter inpatient stay has the highest the RAPT score which is comparable to our study. He found the 
predictive accuracy of $85 \%$ in RAPT score and concluded that those patients who want to be discharge home has higher RAPT score. (OR 9.79, 95\% CI 5.07 to $18.89, P<0.001$ ). The predictive accuracy of our study was $97.4 \%$

Coudeyre et al. [17] studied 134 patients of hip replacement for predictive outcome of surgery in which it was found that RAPT score was useful tool in postoperative decision for patient discharge. The usefulness of preoperative RAPT score in planning patient care postoperatively has limited value because nearly $50 \%$ of the patients has score of 6 to 9 . To increase the accuracy of RAPT score further study is need for 6-9 (intermediate risk group) [13].

Customs, traditions and values play a big role in medical field in our set up and most of the patients that were enrolled were highly cooperative in the postoperative management which has minimized the complications as compared to other studies. There are certain limitations in our study. The sample size is small and only an educated patient who can read and understand the written precautions were included which means that a great chunk of patients was excluded from the study as they are only illiterate. The follow up was very short- and long-term complications were not evaluated.

\section{Conclusion}

Preoperative assessment of the patients undergoing Total Hip Replacement not only reduces complication rates but also reduces socioeconomic burden on hospital as well as on patients. Clinical scoring of a patient that undergo surgery can allow the patient as well as the surgeon to focus on those aspects that need special attention. The preoperative scoring system allows the patients to obtain safe assistance and appropriate care postoperatively.

\section{References}

1. Benedetti MG, Sarti D, Bonfiglioli Stagni S, Marian E (2015) Setting, clinical pathways, fast-track and rehabilitation following primary knee arthroplasty: a literature review. Open Rehabil J 8: 17-24.

2. Monticone M, Ambrosini E, Rocca B, Lorenzon C, Ferrante S, et al. (2014) Task-oriented exercises and early full weight-bearing contribute to improving disability after total hip replacement: A randomized controlled trial. Clin Rehabil 28(7): 658-668.

3. Slover J, Mullaly K, Karia R (2017) The use of the risk assessment and prediction tool in surgical patients in a bundled payment program. Int J Surg 38: 119-122.
4. Oldmeadow LB, McBurney H, Robertson VJ (2003) Predicting risk of extended inpatient rehabilitation after hip or knee arthroplasty. J Arthroplasty 18(6): 775-779.

5. Dauty M, Schmitt X, Menu P, Rousseau B, Dubois C (2012) Using the Risk Assessment and Predictor Tool (RAPT) for patients after total knee replacement surgery. Ann Phys Rehabil Med 55(1): 4-15.

6. Oosting E, Hoogeboom TJ, Appelman-de Vries SA, Swets A, Dronkers JJ, et al. (2016) Preoperative prediction of inpatient recovery of function after total hip arthroplasty using performance-based tests: A prospective cohort study. Disabil Rehabil 38(13): 1243-1249.

7. Mathias S, Nayak US, Isaacs B (1986) Balance in elderly patients: the "get-up and go" test. Arch Phys Med Rehabil 67(6): 387-389.

8. Peters DM, Fritz SL, Krotish DE (2013) Assessing the reliability and validity of a shorter walk test compared with the 10-Meter Walk Test for measurements of gait speed in healthy, older adults. J Geriatr Phys Ther 36(1): 24-30.

9. Wolters U, Wolf T, Stützer H, Schröder T (1996) ASA classification and perioperative variables as predictors of postoperative outcome. Br J Anaesth 77(2): 217-222.

10. Perry M A, Hudson S, Ardis K (2011) If I didn't have anybody, what would I have done?: Experiences of older adults and their discharge home after lower limb orthopaedic surgery. J Rehabil Med 43(10): 916-922.

11. Konopka JF, Hansen VJ, Rubash HE, Freiberg AA (2015) Risk assessment tools used to predict outcomes of total hip and total knee arthroplasty. Orthop Clin North Am 46(3): 351-362.

12. Abbas K, Murtaza G, Umer M, Rashid H, Qadir I (2012) Complications of total hip replacement. J Coll Phys Surg 22 (9): 575-578.

13. Hansen VJ, Gromov K, Lebrun LM, Rubash HE, Malchau H, et al. (2015) Does the risk assessment and prediction tool predict discharge disposition after joint replacement? Clin Orthop Relat Res 473(2): 597601.

14. Bozic KJ, Katz P, Cisternas M, Ono L, Ries MD, Showstack J (2005) Hospital resource utilization for primary and revision total hip arthroplasty. J Bone Joint Surg Am 87(3): 570-576.

15. Bozic KJ, Wagie A, Naessens JM, Berry DJ, Rubash HE (2006) Predictors of discharge to an inpatient extended care facility after total hip or knee arthroplasty. J Arthroplasty 21(6 Suppl 2): 151-156.

16. Tan C, Loo G, Pua YH, Chong HC, Yeo W, et al. (2014) Predicting discharge outcomes after total knee replacement using the Risk Assessment and Predictor Tool. Physiotherapy 100(2): 176-181.

17. Coudeyre E, Eschalier B, Descamps S (2014) Transcultural validation of the Risk Assessment and Predictor Tool (RAPT) to predict discharge outcomes after total hip replacement. Ann Phys Rehabil Med 57(3): 169184.

For possible submissions Click below:

Submit Article 